\title{
Progress with the Jet Experiment
}

\section{P.E. Stott, Abingdon \\ (Jet Joint Untertaking)}

The Joint European Torus (JET) is the largest single project in Europe's coordinated programme of research into the feasibility of nuclear fusion as an energy source. The basic principles of fusion are well known. Energy is released when two light nuclei are fused together to form a heavier one. The most accessible reaction is that between the two heavy isotopes of hydrogen, deuterium and tritium, which fuse to produce an $\alpha$ particle and a neutron:

$$
\mathrm{D}+\mathrm{T} \rightarrow{ }^{4} \mathrm{He}+\mathrm{n}+17.6 \mathrm{MeV}
$$

The cross-section for the two nuclei to fuse together is small (much smaller than for them to scatter elastically off each other) but increases strongly with temperature. Temperatures in the range $10-20 \mathrm{keV}(100-200 \mathrm{MK})$ will be required for fusion and thus the reaction will take place in a fully ionised hot plasma. Clearly the plasma has to be well insulated from contact with material surroundings. The effectiveness of this thermal insulation is conveniently expressed by the energy confinement time $\tau_{E}$ which is defined as the total energy content of the plasma divided by the total power input. The condition for a fusion reactor to become self sustaining is given by the well known Lawson criterion, which for plasma temperatures $T$ in the range 10 to $20 \mathrm{keV}$ is given approximately by

$$
n_{\mathrm{i}} T_{\mathrm{i}} \tau_{E} \geq 3 \times 10^{21} \mathrm{~m}^{-3} \mathrm{keV} \mathrm{s}
$$

(where $n_{\mathrm{i}}$ is the plasma ion density). Thus the product of ion density and confinement time, $n_{i} \tau_{E}$, must exceed about $2 \times 10^{20} \mathrm{~m}^{-3} \mathrm{~s}$.

The most promising magnetic confinement system is the tokamak, which is shown schematically in Fig. 1. The plasma is confined by the poloidal magnetic field generated by a current flowing in the plasma, and stabilised by a much stronger toroidal magnetic field. The resulting magnetic field lines twist helically around the torus, and in an ideal case would form a nested set of toroidal magnetic "surfaces" on which the plasma would be confined.

JET was conceived in the early 1970's when it became clear that progress in this field required the construction of an experiment which would be much larger than the resources of any individual fusion laboratory in Europe. The primary objective of JET was (and is) to bridge the gap between the parameters of existing experiments and those required for a fusion reactor by obtaining and studying a plasma in conditions and with dimensions approaching those needed in a reactor. The construction of the project at a location adjacent to the UK Fusion Laboratory at Culham was approved in mid 1978. The project is a joint venture of 12 European nations (Sweden and Switzerland are members in addition to the EEC countries, Greece joined in 1983, Spain and Portugal have applied

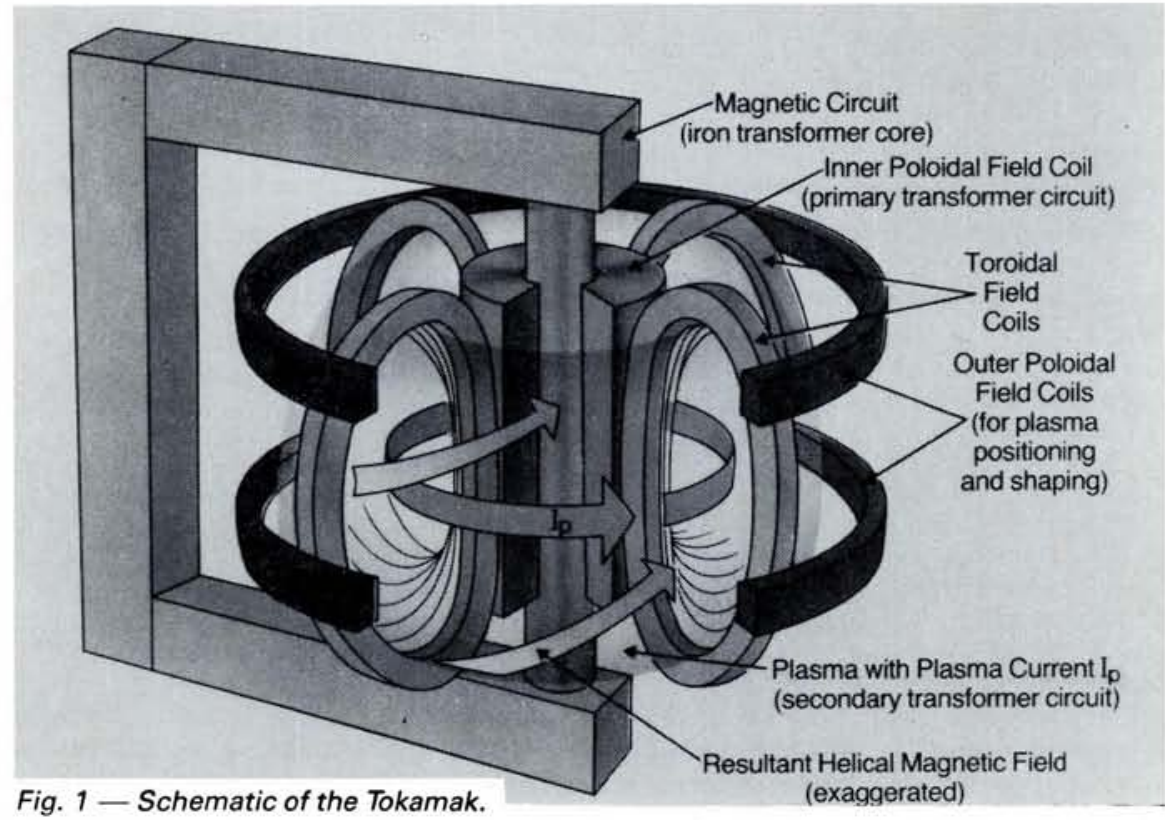

for membership) and the European Atomic Energy Community (EURATOM). The initial agreement covered a 12 year period (1978 to 1990), but discussions are in progress to extend this to 1992. The total cost of the project over the 12 year period is about $1000 \mathrm{M} \mathrm{ECU}$.

The first operation of JET (Fig. 2) took place on schedule in mid 1983 and the experiment is now the largest of three large tokamaks operating in the world (TFTR at Princeton in the USA started operating in December 1982, and JT-60 at JAERI in Japan started operating in April 1985). The relative parameters of these three experiments are illustrated in Fig. 3. The three experiments are complementary and there is close collaboration between the projects.

\section{Ohmically Heated Plasmas}

JET has now been operating for three years and there has been a steady progress in improving the plasma operating conditions. Discharges are now run routinely at the full design value of the toroidal magnetic field $B_{T}=3.4 \mathrm{~T}$ and with discharge currents up to $5 \mathrm{MA}$ which actually exceeds the design value of 4.8 MA by a small margin. Experiments until 1985 concentrated on plasmas heated ohmically, that is by the resistive heating of the plasma current itself. Systematic studies have been made of the dependence of the energy confinement time $\tau_{E}$ on various plasma parameters. There is general agreement with data extrapolated from smaller experiments and in particular with the large American tokamak TFTR (discharges have been run in JET with the same dimensions and other parameters as TFTR). The general trend of these data is that in ohmic discharges the confinement improves with increasing plasma density $n_{\mathrm{e}}$ and the parameter $q_{a}=5 a b B_{\mathrm{T}} / R I_{\mathrm{p}}$ (where $I_{\mathrm{p}}$ is the plasma current in MA, $B_{T}$ the toroidal magnetic field in Tesla, $R$ is the major radius of the discharge in metres and $a$ and $b$ are the horizontal and vertical minor radii) which is a measure of the helicity of the magnetic field lines at the edge of the plasma. However the JET data show a much weaker dependence on $n_{e}$ and $q_{a}$ than in smaller tokamaks (typically in JET, $\tau_{E} \sim n_{\mathrm{e}}{ }^{0.4} q_{a}{ }^{0.3}$ ) and at higher densities the density dependence saturates completely. There is a strong 
dependence of confinement time on the plasma dimensions with $\tau_{E} \sim R^{1.7} a^{1.3}$. Thus if the aspect ratio $R / a$ and other parameters remained constant, a tokamak twice as large as JET would have eight times the confinement. There does not seem to be any explicit dependence of the confinement time on the elongation, except that which comes implicitly through $q_{a}$. The best value of confinement time $\tau_{E} \cong 0.8 \mathrm{~s}$, has been reached in an ohmic discharge $\left(I_{\mathrm{p}}=3 \mathrm{MA}, B_{\mathrm{T}}=\right.$ 3.4 T). The highest ion and electron temperatures are about $3 \mathrm{keV}$ and $4 \mathrm{keV}$ respectively and the average electron density is about $4 \times 10^{19} \mathrm{~m}^{-3}$.

\section{Additional Heating}

In tokamaks, ohmic heating becomes less effective as the plasma temperature rises and the resistivity falls. The maximum temperature obtainable in JET by ohmic heating is about $\sim 4 \mathrm{keV}$. To reach higher temperatures, JET has two systems for additional heating.

Radio frequency heating utilises electromagnetic waves launched from large antennae just outside the edge of the plasma. Frequencies in the range 25 to $50 \mathrm{MHz}$ are used which heat the plasma via the gyro-frequency resonances of $\mathrm{H}^{+}$and ${ }^{3} \mathrm{He}^{++}$ions which are added to the deuterium plasma in small amounts (typically $<10 \%$ ). The position of the resonance layer, and thus the power deposition profile, can be varied either by changing the toroidal magnetic field or the frequency of the heating wave. During 1985 , radio frequency heating experiments were started in JET using two antennae which could be connected in different configurations (monopole, dipole and octopole) to study how the coupling efficiency varied with the distance between the plasma edge and the antennae. The maximum power coupled into the plasma was $\cong 5 \mathrm{MW}$ for $2 \mathrm{~s}$. A third antennae was added at the start of 1986 allowing the coupled power to be raised to $\cong 7 \mathrm{MW}$. The maximum central electron temperature which has been achieved is around $7.5 \mathrm{keV}$.

The second type of additional heating system installed on JET uses powerful neutral atom beams (P. Lomas, Europhys. News 16 (1985) 4). The beams are produced by accelerating ion beams to energies of some $80 \mathrm{keV}$. After neutralisation, the neutral beams penetrate the magnetically confined plasma, and become ionised by collisions. The resulting fast ions are trapped by the tokamak magnetic fields, and transfer their energy by collisions to the background plasma. Early in 1986, neutral injection heating experiments started in JET with a sys-

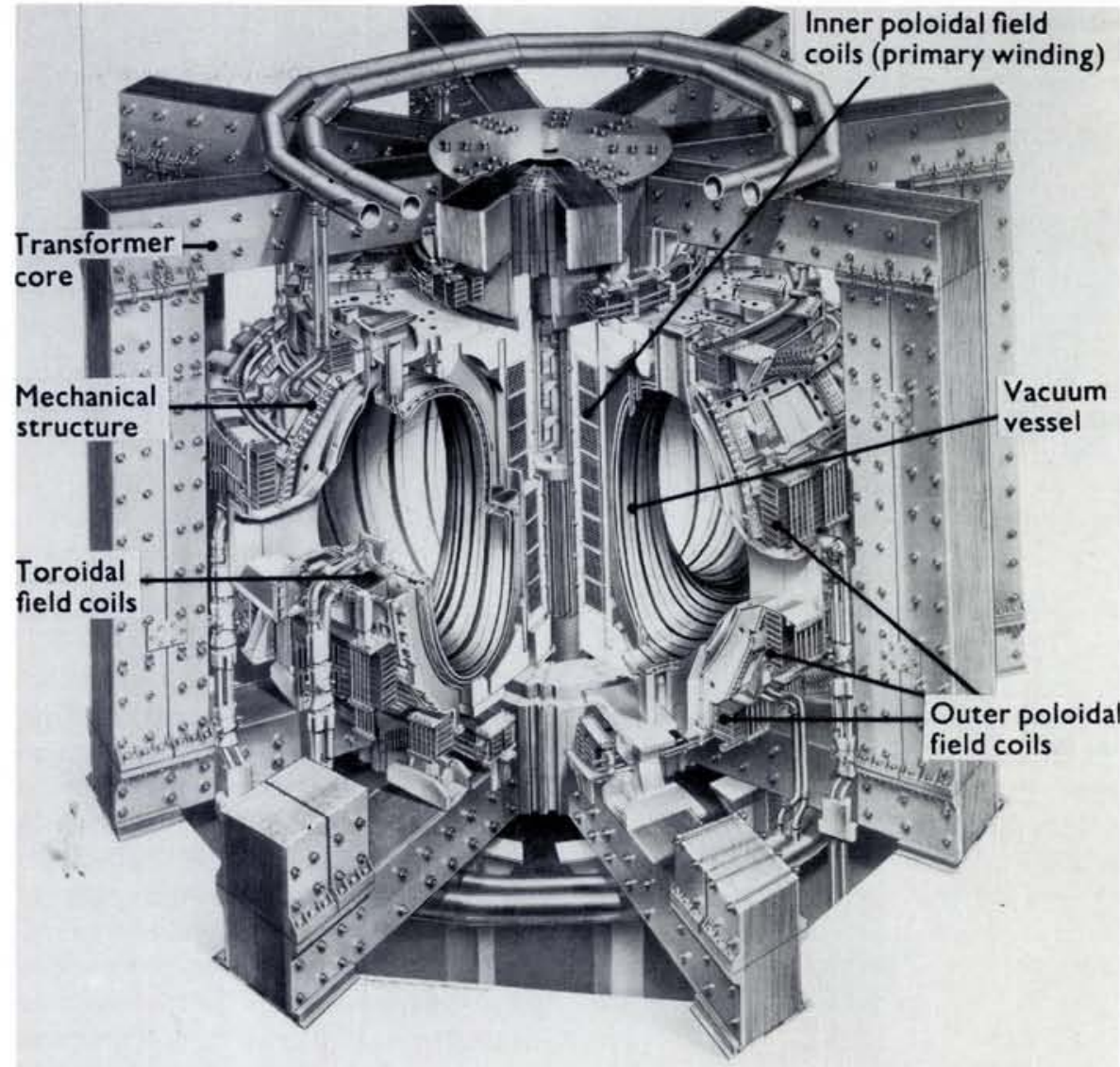

Fig. 2 - Schematic of the JET device. The vertical height of the transformer limbs is $12 \mathrm{~m}$.

tem capable of injecting up to $\cong 6 \mathrm{MW}$ of $80 \mathrm{keV} \mathrm{H}{ }^{\circ}$ or $\cong 9 \mathrm{MW}$ of $80 \mathrm{keV} \mathrm{D}^{\circ}$ into the plasma. The maximum plasma temperature obtainable with neutral beam heating depends on the plasma density. At medium to high densities, the ions have been heated to about $6.5 \mathrm{keV}$ and the electrons to $4.8 \mathrm{keV}$ by $5.5 \mathrm{MW}$ of neutral beams. At lower densities, higher ion temperatures in excess of 10 $\mathrm{keV}$ have been produced in recent experiments.

The global energy confinement times with both additional heating systems are shorter than in the ohmically heated target plasma. This confirms experience in other experiments that confinement generally degrades as the heating power is increased. It appears that this degradation depends primarily on the total power input to the plasma - i.e. it is independent of the type of heating (radio frequency, neutral beam or ohmic), and at least to first order, it is independent of whether the power is deposited close to the centre of the plasma or closer to the edge. There have been attempts to fit empirical scaling laws to the measured energy confinement times, but the range of data so far obtained in JET does not allow us to distinguish clearly whether the confinement will continually degrade as the power is increased (i.e. $\tau_{E} \sim$ const $P^{-\alpha}$ with $\alpha \cong 0.5$ ) or whether

Fig. 3 - Comparison of the dimensions and other parameters of the three large tokamaks now in operation.

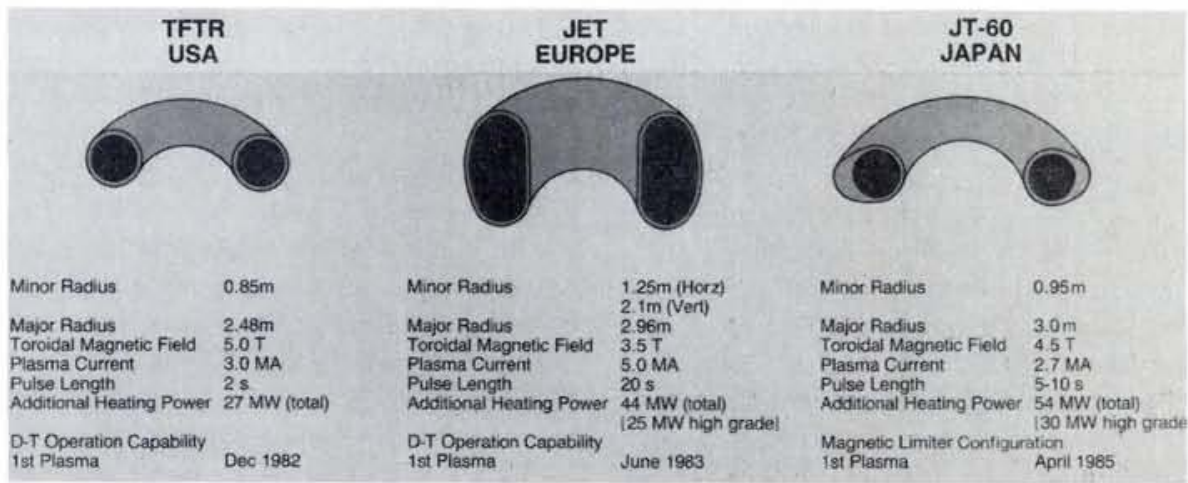




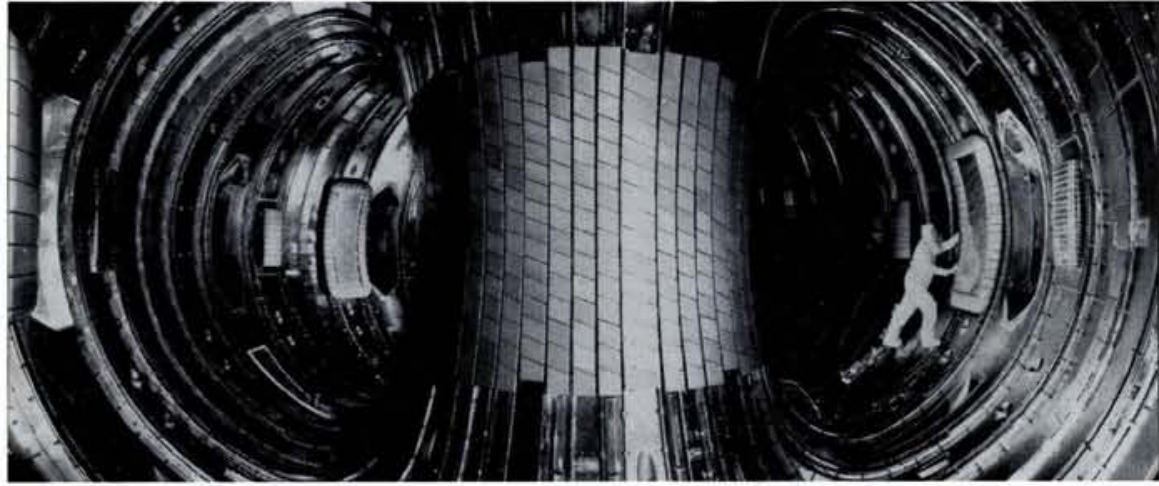

Fig. 4 - Composite view inside the toroidal vacuum vessel. The carbon protective tiles can be seen on the inner wall. The large panel being adjusted is one of the antennae for radio frequency heating. The smaller panels either side of the antenna are limiters.

the confinement approaches an asymptotic value at higher heating powers (i.e. $\left.\tau_{E} \sim a+b / P\right)$. Although there are no striking differences in the effect on confinement of the different additional heating methods, there are some differences in the effects on other plasma properties (e.g. purity and maximum density) as will be discussed below. We have also seen some interesting synergistic effects when the radio frequency heating interacts with the fast injected beam ions.

\section{Plasma Purity}

The JET vacuum vessel is fabricated out of inconel (mainly nickel and chromium) and is usually operated at a temperature $>300^{\circ} \mathrm{C}$. The inner wall is covered with carbon tiles (see Fig. 4) and there are now eight carbon limiters $(0.8 \times 0.4 \mathrm{~m})$ on the mid-plane at the outer major radius. The discharge can be positioned radially so that it is either in contact with the outer limiters, or with the inner wall.

The radio frequency heating antennae are in similar positions to the limiters, but slightly further away from the edge of the plasma and are protected by carbon tiles. As in all tokamaks, the vacuum vessel has to be conditioned to remove lightly absorbed surface contaminants before reliable tokamak discharges can be obtained. The usual procedure in JET uses a continuous glow discharge in hydrogen or deuterium.

The main impurities are carbon $12-4 \%$ of the plasma electron density) and oxygen $(1-2 \%)$ which enter the discharge in roughly equal amounts from the walls and limiters. These low $Z$ impurities become fully ionised before reaching the centre of the plasma, and hence they cool the edge but not the central core of the plasma by radiation. The most important metallic impurity is nickel and the concentration varies over the range $0.001-0.3 \%$ depending on the plasma conditions. The original sources of the nickel are the inconel walls and the nickel screens on the RF antennae, but the nickel from the walls enters the plasma via the carbon limiters in a two stage process. The limiters become covered in metal during glow cleaning and following discharges which have ended with disruptions (see below), then during normal tokamak discharges the metal is eroded from the limiter and enters the plasma. Some nickel is also observed to enter the plasma from the RF antennae when these are operated. However, the metallic impurities in JET are generally not important and do not significantly cool the plasma.

During the later part of 1984 and throughout 1985 carbon was routinely deposited onto the surfaces of the vacuum vessel by adding a small concentration $(2-17 \%)$ of methane to the hydrogen or deuterium gas used for glow cleaning. This procedure was observed to reduce the concentrations of metals in the plasma on subsequent discharges, most probably by burying existing metallic deposits on the limiters under a layer of fresh carbon. However the beneficial effects, which lasted usually for about 20 discharges, were accompanied by difficulties in controlling the plasma density, and this procedure has been discontinued (except on one occa-

The large area of carbon on the inside wall has a strong pumping effect on the plasma, and the plasma density can be controlled by positioning the discharge in contact with the inside wall rather than on the main limiters.

\section{Disruptions}

All tokamaks are observed to become unstable when either the current or density are increased beyond certain limits. Usually these instabilities start to grow within the plasma in the region where the helicity of the magnetic field lines (denoted by the parameter $q$ ) is rational, i.e. where individual field lines close on sion) during 1986. themselves after $q$ transits around the torus. Usually the instability grows on the field lines with $q=2$, spreads to neighbouring rational field lines $(q=3 / 2$ etc.) and ultimately the discharge is terminated in a catastrophic collapse, which is called a disruption.

The overall stability of the discharge depends on $q_{a}$ which is the helicity at the edge of the discharge and on the mean plasma density $n_{\mathrm{e}}$. Note that $q_{a}$ is inversely proportional to plasma current. The limit in high current operation in JET is found when $q_{a} \sim 2$. For a plasma with the maximum toroidal field $B_{\mathrm{T}}=3.4 \mathrm{~T}$ and poloidal cross-section $1.2 \times 1.9 \mathrm{~m}$ this will allow currents up to $7 \mathrm{MA}$. At higher values of $q_{a}$, (i.e. lower discharge currents) disruptions occur when the plasma density is raised too high. For ohmic discharges the limiting density is given by the empirical expression $n_{\mathrm{e}} \leq$ $12 B_{\mathrm{T}} / R q_{\mathrm{a}} \times 10^{19} \mathrm{~m}^{-3}$ where $B_{\mathrm{T}}$ is measured in tesla and $R$ in metres. In JET it is usually observed that the total power lost by radiation increases as the plasma density is raised, and becomes equal to the total power input when the plasma disrupts. This is consistent with one model of disruptions whereby radiative cooling of the plasma edge causes a shrinkage of the plasma current profile which drives the plasma unstable. Clearly the density limit should be increased either by reducing the radiation losses (i.e. making the plasma cleaner) or by increasing the power input and both effects are generally seen in tokamaks. The density limit in JET has been raised by a factor $\cong 2$ with a neutral injection heating power input of $\cong 8 \mathrm{MW}$, but similar improvements in density are not observed with radio frequency heating. This is not completely understood; it may be due to the fact that the radio frequency heating brings into the plasma additional metallic impurities, or it may be that the neutral beam raises the density by depositing particles deeper into the plasma.

\section{Sawteeth}

The temperature and density of the plasma in the core of tokamaks usually fluctuate periodically in time. These fluctuations are called "sawteeth" which describes the relatively slow linear growth followed by a rapid collapse. Sawteeth had been extensively studied in earlier tokamaks and were generally claimed to be in good agreement with existing theory. The sawteeth in JET were found to have quite different characteristics, many of which could not be explained by existing theory. The principal features of the JET sawteeth are that 
the rise phase of temperature and density can be very long, a duration of several tenths of a second are usual in ohmically heated plasmas, and a duration of more than one second has been observed in plasmas with additional heating. During this rise phase, there may be one or more partial collapses which affect only the plasma at the outer part of the core, but not that on the axis. The final collapse often occurs spontaneously without the precursor activity usually seen in other experiments, and takes place on a time scale much shorter ( $\cong 100 \mu$ s) than predicted by existing theory. Similar phenomena are now being reported from other tokamak experiments.

\section{Magnetic Separatrix}

The goal of increasing the quality of confinement in tokamaks has led to a renewed interest in the "divertor" or "magnetic limiter" configuration in which the plasma boundary is defined by a magnetic separatrix, detached from material limiters and the vacuum vessel. Such configurations may afford improved thermal insulation of the plasma as well as reduced contamination from impurities. The region at the plasma boundary may be able to sustain a high edge temperature with a larger temperature gradient when substantial additional heating is applied. In some tokamaks, these so-called " $\mathrm{H}$ mode" discharges show improved energy confinement with additional heating compared to usual "L mode" ones (F. Wagner, Europhys. News 17 (1986) 4).

The formation of a magnetic separatrix has been shown to be possible in JET by suitable programming of the currents in the coils which drive the discharge current and shape the plasma. In preliminary experiments separatrix configurations, with either one or two null points at the top or bottom of the discharge, have been established and maintained for several seconds in discharges with plasma currents up to $3 \mathrm{MA}$. The presence of these null points in the poloidal field some $10-15 \mathrm{~cm}$ inside the vacuum vessel is shown by calculations of the field boundary from magnetic pickup coil measurements and the plasma is apparently well detached from the limiter and inner vessel wall. This is confirmed by the absence of the plasma light emission from these surfaces at the time of separatrix formation. The area of intersection of the plasma at the separatrix points with the vacuum vessel wall was observed with an infra-red camera, and the change in field line connection length was observed with a Langmuir probe. Experiments are now in progress to apply powerful additional heating to these configurations to study their confinement properties. The preliminary results show that the confinement is about double that of normal discharges. A typical value of the Lawson product $n_{\mathrm{i}} T_{\mathrm{i}} \tau_{\mathrm{i}}$ is $2 \times 10^{20} \mathrm{~m}^{-3} \mathrm{~s} \mathrm{keV} \mathrm{with} I_{\mathrm{p}}=3$ $\mathrm{MA}$ and $10 \mathrm{MW}$ of neutral beam heating.

\section{Future Plans}

The present programme for JET includes a substantial increase in the additional heating power during the next three years. The Ion Cyclotron Resonant Heating power will be increased progressively to $15 \mathrm{MW}$ for 20 s in 1988. A second neutral beam line will be installed in 1987 bringing the total neutral beam power to $\cong 10 \mathrm{MW}$ at $80 \mathrm{keV} \mathrm{H}^{\circ}$ or $160 \mathrm{keV} \mathrm{D}^{\circ}$ or $\cong 18 \mathrm{MW}$ at $80 \mathrm{keV} \mathrm{D}^{\circ}$.

The key quantity in achieving fusion is the product of density, temperature and energy confinement time $n_{i} T_{i} \tau_{E}$. The best value obtained so far in JET is $\cong 2$ $\times 10^{20} \mathrm{~m}^{-3} \mathrm{keV}$ s compared with a value of $7 \times 10^{20}$ which would give significant $\alpha$ particle heating or a value $>3 \times$ $10^{21}$ which would be needed for JET to become self sustaining.

However it is clear from JET and other experiments that the degradation of energy confinement which is observed with increasing heating power is a serious and fundamental problem. Thus whilst with sufficient additional heating power we can attain the central ion temperatures $T_{i} \cong 20 \mathrm{keV}$ required for fusion this will be offset by the concomitant degradation of the energy confinement. Recent results with the magnetic separatrix configurations suggest that regimes of good confinement can be found. A number of ideas which may improve the confinement are being considered by the JET team with a view to implementing some or all of these schemes over the next few years. The ideas presently under study include control of sawteeth, control of temperature and/or current profiles, operation with an internal magnetic separatrix, operation on the inner wall and pellet refuelling. The problem of ion depletion by impurities is also serious in that it reduces the $n_{i} T_{i} \tau_{i}$ product by a factor of about two, and we are studying pellet refuelling and operating with beryllium limiters as possible solutions. The main thrust of JET's programme over the next few years will be towards improving the confinement properties of discharges with powerful neutral beam and radio frequency heating. These experiments will be conducted in hydrogen and deuterium in order to minimise neutron production, but the experiment is being prepared for operation with tritium in 1991-92.

\section{BIBLIOGRAPHY}

1. Rebut P.H. et al., "Design, Construction and First Operational Experience on the Joint European Torus (JET)", Special edition of Fusion Technology, Jan. 1987.

2. Gibson A., "The Scientific Programme of JET", Europhys. News 14 (1983) 4.

3. Bickerton R.J., "Latest Results from JET", Plasma Physics and Controlled Fusion 28 (1986) 55.

4. Lallia P.P. et al., "Plasma Heating in JET", Plasma Physics and Controlled Fusion 28 (1986) 1211.

5. Engelhardt W. et al., "Impurity behaviour in JET", Plasma Physics and Controlled Fusion 28 (1986) 1401.

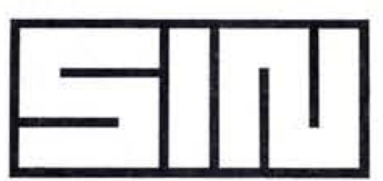

\section{Theoretical Physicists}

Two permanent positions for theoretical physicists are open at SIN, the Swiss Institute for Nuclear Research, Switzerland. Substantial experience in the field of strong and/or electroweak interactions is required. The physics orientation must be relevant to the experimental programme carried out at SIN which is based on a $600 \mathrm{MeV}$ proton accelerator with intensive secondary beams of pions and muons. Close contact with experimental groups working at SIN is expected. Present experiments are concerned with weak and forbidden decays of pions and muons, mesic atoms, pion and nucleon induced reactions, and with applications of the muon spin rotation technique to problems in condensed matter physics. Further information may be obtained from

Prof. Milan P. Locher (Telephone: (56) 993656 or 9936 65).

The usual documentation including the names and addresses of three referees should be sent by 31 January, 1987 to:

SIN, Swiss Institute for Nuclear Research, Personnel Dept., CH - 5234 Villigen/Switzerland, Code 38. 